\title{
Modulare endoprothetische Rekonstruktion bei malignen Knochentumoren
}

\author{
Ralf Dieckmann, Arne Streitbürger, Maurice Balke, Marcel-Philipp Henrichs, \\ Markus Nottrott, Georg Gosheger, Jendrik Hardes
}

\section{Zusammenfassung}

Extremitätenerhaltende Chirurgie mit modularen Tumorendoprothesen ist heutzutage in den meisten Fällen erfolgreich möglich. Fast jedes Gelenk, sogar ganze Knochen (wie z.B. ein totaler Femur oder Humerus) können mit modularen Endoprothesensystemen rekonstruiert werden. Schwerwiegende Komplikationen sind das Lokalrezidiv (1-9\%), die periprothetische Infektion (5-36\%) mit dem Risiko der sekundären Amputation und die aseptische Lockerung (5-27\%). Im Gegensatz dazu ist der Polyethylenverschleiß bei jungen sportlichen $\mathrm{Pa}$ tienten als unvermeidlich anzusehen. Obwohl die Komplikationsraten bei Tumormegaendoprothesen deutlich gesunken sind, sind sie im Vergleich zu der primären Endoprothetik immer noch deutlich höher. In diesem Artikel beschreiben wir das modulare Tumorendoprothesensystem, das in unserer Klinik entwickelt wurde, geben eine Übersicht über das postoperative Management und diskutieren die Indikationen, die Limits und Komplikationen wie auch die funktionellen Ergebnisse.

\section{Modular Endoprosthetic Reconstruction in Cases of Malignant Bone Tumours}

Limb salvage with modular megaendoprosthesis is well established today. Almost every joint and even the entire bone (e.g., complete femur or humerus) can be replaced using modern modular endoprostheses. The major complications are local recurrence (1$9 \%)$, periprosthetic infections with the risk of secondary amputation (5-36\%) and aseptic stem loosening (5-27\%). Unavoidable are breakages or abrasions of the polyethylene components in young active patients. Although the complication rates are decreasing, revision surgery is still higher than in primary joint arthroplasty. In this review we present the modular endoprosthesis system that has been developed in our institution, summarise the postoperative management, and discuss the indications, limits and complications as well as the functional results.

\section{Einleitung}

Heutzutage kann in den meisten Fällen bei Patienten mit malignen Knochentumoren ein extremitätenerhaltendes Operationsverfahren mit weiten Resektionsgrenzen angewendet werden [1]. Nur in seltenen Fällen ist eine Amputation oder Umdrehplastik aus onkologischen Gründen erforderlich. Die Verwendung von Tumormegaendoprothesen hat sich in den letzten Jahrzehnten zur Rekonstruk-

OP-JOURNAL 2010; 26: 186-190

(c) Georg Thieme Verlag KG Stuttgart · New York DOI http://dx.doi.org/10.1055/s-0030-1250558 tion von großen Knochendefekten nach Tumorresektionen etabliert. Ein Nachteil in Bezug auf ein Lokalrezidiv oder Überleben hat sich nicht gezeigt [2]. Während in den Anfängen der Tumorprothesenära nur individuell gefertigte Tumormegaendoprothesen verfügbar waren, können heutzutage moderne modulare Tumorprothesensysteme als Goldstandard verwendet werden [2].

Individualanfertigungen waren sehr kostenintensiv und zeitaufwendig, sodass es in manchen Fällen zu einer verzögerten Lokaltherapie und sogar zu einer Verschlechterung des Überlebens geführt hat. Mit modernen modularen Implanta- ten können auch extrem große Knochendefekte individuell mit guten Ergebnissen schnell und kostensparend rekonstruiert werden [1].

In den letzten 30 Jahren konnte die 5jährige Standzeit von Tumormegaendoprothesen von 20 auf $85 \%$ verbessert werden, obwohl die Patienten meistens jung und aktiv sind. Dennoch ist die Komplikationsrate höher als im Bereich der primären Endoprothetik [3].

Im Folgenden berichten wir über unsere Erfahrung mit dem von uns verwendeten modularen Tumormegaendoprothesensystem (Abb. 1), beschreiben das postoperative Behandlungskonzept, erläutern die Indikationen, die typischen Komplikationen und die Grenzen.

Bei Patienten mit malignen Knochentumoren kann in den meisten Fällen ein extremitätenerhaltendes Operationsverfahren mit weiten Resektionsgrenzen angewendet werden.

\section{Chirurgische Technik}

Mithilfe des modularen universalen Tumor- und Revisionssystems (MUTARS, Fa. Implantcast, Buxtehude) können große Knochendefekte sowohl der oberen als auch der unteren Extremität erfolgreich rekonstruiert werden [1].

Das modulare Design erlaubt es, Knochendefekte in 1-cm-Schritten auszugleichen und eine Torsionsanpassung in $5^{\circ}$-Schritten durchzuführen. Die verschiedenen Komponenten werden mit einer Schraube intern fixiert (Abb. 1).

Meistens werden Tumorprothesen zur Rekonstruktion des proximalen und distalen Femurs, der proximalen Tibia und des proximalen Humerus verwendet. Selbst die Rekonstruktion des gesamten Femurs und Humerus mit angrenzenden Gelenken ist mit diesem System ohne Sonderanfertigung möglich [1]. 


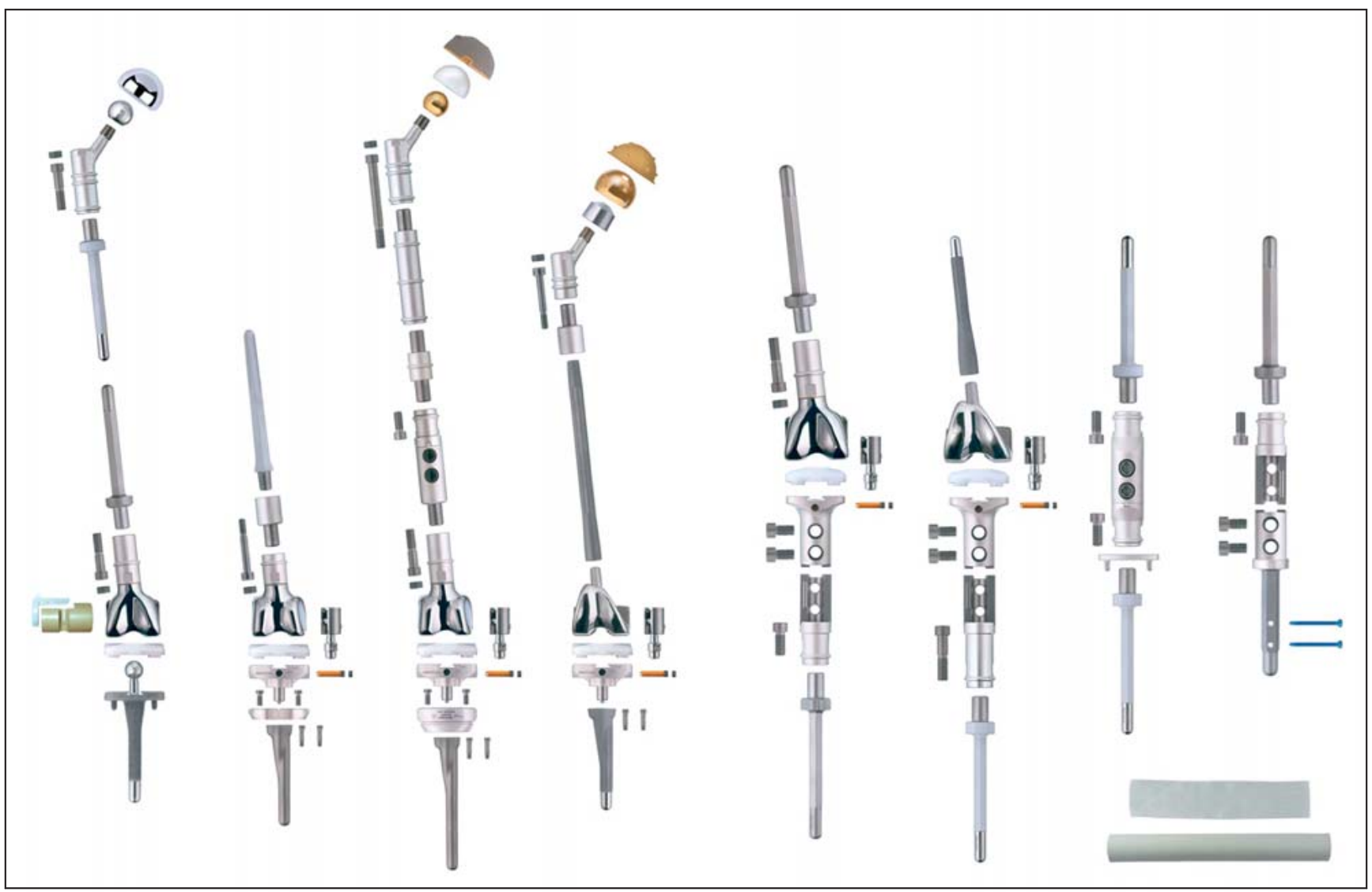

Abb. 1 Modulares universales Tumor- und Revisionssystem (MUTARS) zur Rekonstruktion der unteren Extremität. Die Komponenten werden jeweils durch Schrauben verbunden.

Heutzutage wird in den meisten Fällen ein zementfreier hydroxyapatitbeschichteter Schaft verwendet. Der Schaft hat ein hexagonales Design, das zu einer hervorragenden primären Rotationsstabilität führt. Die genaue Planung erfolgt an einem digitalen Röntgenbild. Der Standardschaft im Bereich der unteren Extremität hat eine Länge von $12 \mathrm{~cm}$ und sollte aus biomechanischen Gründen einen Mindestdurchmesser von $12 \mathrm{~mm}$ haben.

Eine zementierte Verankerung kommt bei älteren Patienten (> 65 Jahren), Patienten mit ausgeprägter Osteopenie oder Osteoporose, z.B. nach prolongierter präoperativer Immobilisation bei Chemotherapie, oder bei Verankerungen in der Metadiaphyse, wo eine zementfreie Verankerung aufgrund der anatomischen Verhältnisse nicht möglich ist, zur Anwendung.

Der Kniegelenksmechanismus ist entweder mit einem „rotating hinge“-Mechanismus mit dem PEEK-Optima-Schloss oder einem „metal on metal“-Mechanismus verbunden. Zwischen dem tibialen und femoralen Anteil artikulieren die Gelenkflächen auf einem Polyethyleninlay.
Die Refixation von Muskeln und Sehnen (z.B. der Glutealmuskulatur im Bereich des proximalen Femurs, der Patellasehne im Bereich der proximalen Tibia oder der Rotatorenmanschette im Bereich des proximalen Humerus) erfolgt mithilfe eines MUTARS-Anbindungsschlauchs, der an der Tumorprothese befestigt wird [1].

Neben der Refixation von Muskulatur eignet sich der Anbindungsschlauch für die Rekonstruktion einer Kapsel, z.B. beim proximalen Femurersatz (Abb. 2). In Kombination mit einem Duokopf treten Luxationen nur selten auf [4].

Tumorprothesen werden zur Rekonstruktion des proximalen und distalen Femurs, der proximalen Tibia, des proximalen Humerus und des gesamten Femurs und Humerus angewendet.

\section{Postoperatives Managment}

Postoperativ erfolgt in unserer Klinik bei allen Patienten eine prophylaktische parenterale Antibiotikatherapie (z.B. ein 3.-Generations-Cephalosporin) für $3 \mathrm{Ta}-$ ge, gefolgt von einer oralen Antibiose bis zum Abschluss der Wundheilung. Ande- re Kliniken führen eine Single-Shot-Antibiose durch. Des Weiteren werden alle Patienten präoperativ darüber informiert, dass eine antibiotische Prophylaxe bei möglichen systemischen Bakteriämien, z.B. bei einer schweren allgemeinen Infektion, einer Tonsillitis oder einer Zahnbehandlung, erforderlich ist, um periprothetische hämatogene Infektionen $\mathrm{zu}$ vermeiden.

Bei Patienten mit einem proximalen $\mathrm{Hu}-$ merusersatz wird das Schultergelenk für 4-6 Wochen in einem Gilchrist-Verband immobilisiert. Ellenbogengelenk und Handgelenk werden ständig physiotherapeutisch behandelt, um Kontrakturen und Lymphödeme zu vermeiden.

Im Falle einer zementfreien Versorgung im Bereich der unteren Extremität belasten die Patienten mit $10 \mathrm{~kg}$ für 6 Wochen, danach erfolgt eine schrittweise Steigerung der Belastung über einen Zeitraum von 4-6 Wochen. Patienten mit proximalem Femurersatz mit Duokopfversorgung können sich postoperativ frei bewegen. Bei Patienten mit einer Pfannenversorgung ist, je nach Weichteilsituation, eine Immobilisation bis $\mathrm{zu}$ 
4 Wochen im Bett in Erwägung zu ziehen, um das Luxationsrisiko durch eine Weichteilkonsolidierung zu reduzieren. Heutzutage verwenden wir in Kombination mit dem proximalen Femurersatz eine tripolare Pfanne. Durch den Einsatz der tripolaren Pfanne konnte die Luxationsrate deutlich reduziert werden.

Bei Patienten mit einem distalen Femurersatz ist eine Bewegungslimitierung nur im Falle eines Gastroknemius-Schwenklappens erforderlich (4 Wochen Ruhigstellung in Extension). Hingegen ist bei Patienten mit einem proximalen Tibiaersatz (Abb. 3) eine Ruhigstellung in Extension essenziell, da die Patellasehne am Anbindungsschlauch anwachsen muss. Eine Ruhigstellung erfolgt in der Regel mit einer Knieorthese. Nach der Ruhigstellung kann direkt eine Flexion bis maximal $90^{\circ}$ erfolgen. Eine Beugung darüber hinaus sollte vermieden werden, da ansonsten ein erheblich größerer Verschleiß des Polyethyleninlays erfolgt.

Bei einem notwendigen Mesh-Graft zum spannungsfreien Hautverschluss über einem Muskelschwenklappen, meistens bei Patienten mit einem proximalen Tibiaersatz, lassen sich mithilfe einer Vakuumtherapie für 5 Tage hervorragende Ergebnisse erzielen.

\section{Komplikationen}

Ein Lokalrezidiv ist die schwerwiegendste Komplikation der extremitätenerhaltenden Chirurgie, vergesellschaftet mit einer deutlichen Verschlechterung der Prognose [5]. In der Literatur ist eine Lokalrezidivrate zwischen 1 und 9\% beschrieben und eine ablative Therapie meistens erforderlich. Daher ist ein weiter Resektionsabstand nach Enneking unbedingt erforderlich $[1,3,4]$.

Ein Lokalrezidiv ist die schwerwiegendste Komplikation der extremitätenerhaltenden Chirurgie und hat in den meisten Fällen eine Amputation zur Folge.

Die 5-10 Jahre Standzeit für moderne Tumormegaendoprothesen liegt zwischen 69 und 90\% [1-3]. Aufgrund der Biomechanik haben Prothesen der oberen Extremität und des proximalen Femurs eine höhere Standzeit als Prothesen im Bereich des Kniegelenks [2,3].

Bei den Prothesenkomplikationen ist zwischen großen Revisionen, wie periprothetischen Infektionen, Luxationen, aspetischen Schaftlockerungen und Frak-
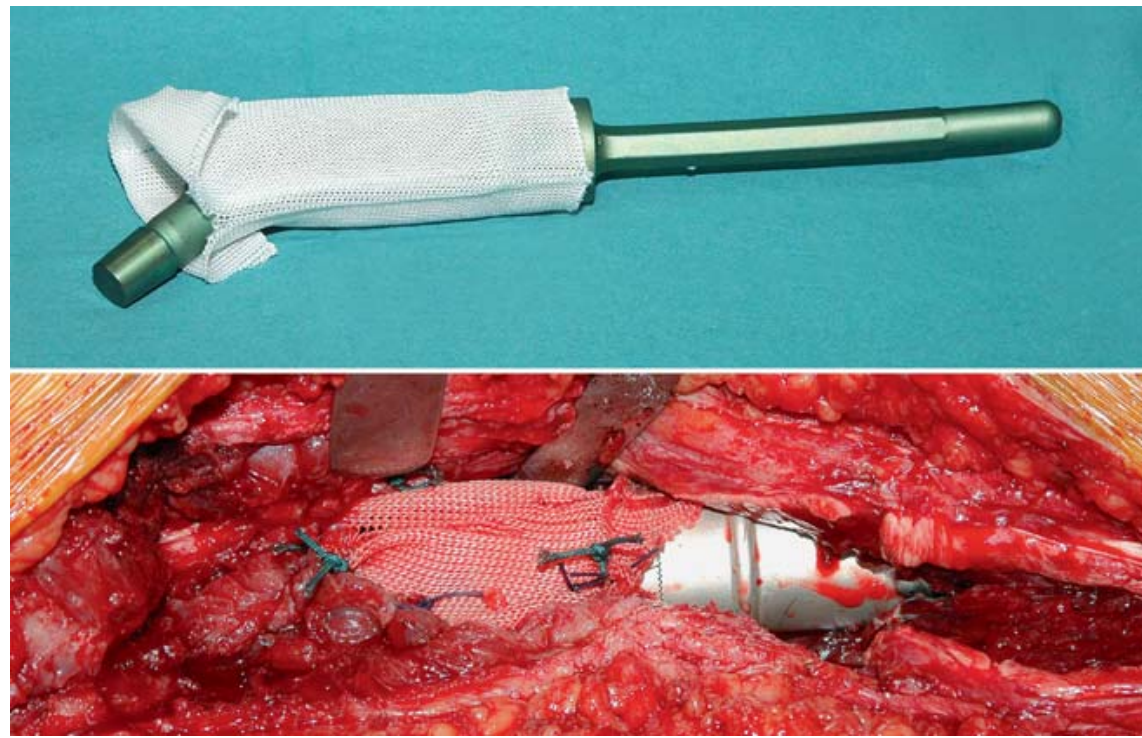

Abb. 2 MUTARS-Anbindungsschlauch. Der Anbindungsschlauch, hier am Beispiel einer proximalen Femurrekonstruktion, wird mit nicht resorbierbaren Fäden am Prothesenkorpus fixiert und mit der umliegenden Muskulatur und Kapselresten vernäht.

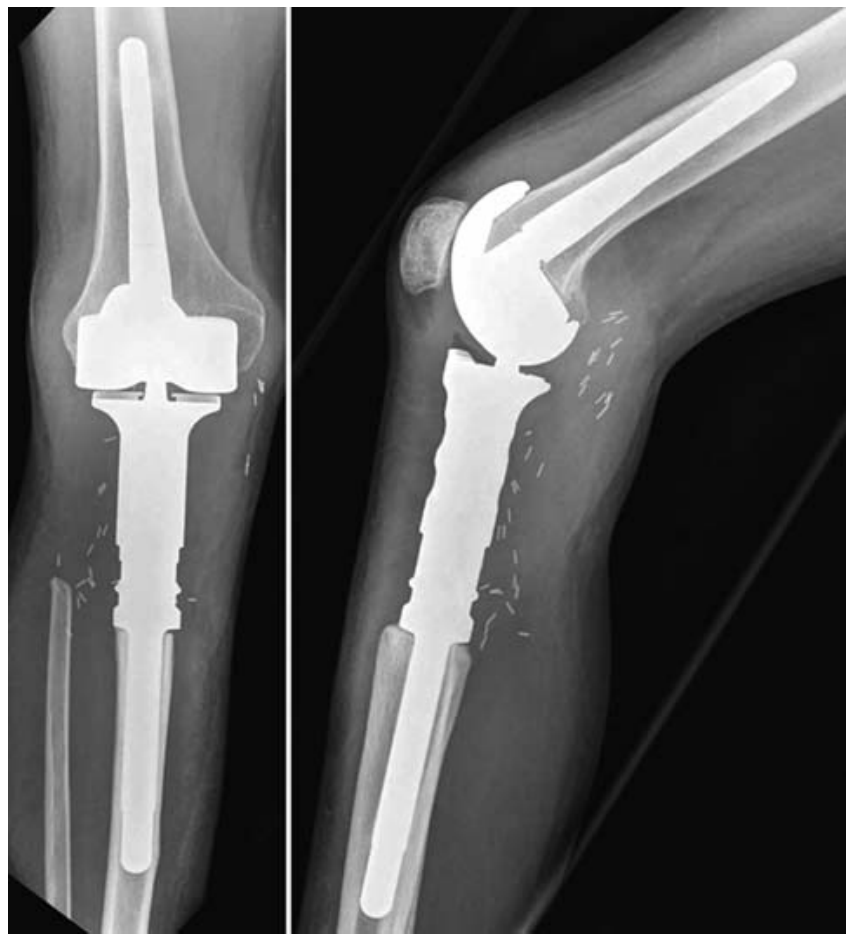

Abb. 3 Postoperatives radiologisches Ergebnis einer proximalen Tibiarekonstruktion mit einer MUTARS-Prothese.

turen, und kleinen Revisionen, wie z.B. dem Polyethylenverschleiß und der oberflächlichen Wundheilungsstörung, zu unterscheiden.

Große Komplikationen sind die periprothetische Infektion, die Luxation, die aseptische Schaftlockerung und Frakturen; kleine Komplikationen sind Polyethylenverschleiß und oberflächliche Wundheilungsstörungen.
Die periprothetische Infektion ist neben dem Lokalrezidiv die schwerste Komplikation bei Tumormegaendoprothesen. In der Literatur variieren die Infektionsraten zwischen 5 und 36\%, wobei im Bereich der oberen Extremität die niedrigsten und im Bereich der proximalen Tibia die höchsten Infektionsraten beschrieben sind $[1,3,6]$. Im Fall einer periprothetischen Infektion ist zumeist ein 2-zeitiger Prothesenwechsel erforderlich. Eine Explantation der Prothese und 
Implantation eines antibiotikahaltigen Platzhalters ist unumgänglich. Erst nach Ausheilung der Infektion ist die Replantation einer Tumormegaendoprothese möglich [6]. Nur im Fall eines Frühinfekts ist eine Revision mit Débridement, JetLavage und Wechsel der Kunststoffteile möglich $[1,3,6]$. Im Falle einer periprothetischen Infektion kann es zu einer sekundären Amputation der betroffenen Extremität (19-46\%) aufgrund einer Infektpersistenz kommen [6]. Mithilfe der Silberbeschichtung der MUTARS-Prothese konnten beim proximalen Femur- und Tibiaersatz die Infektionsrate auf 5,9\% reduziert und Amputationen vermieden werden [7].

Eine aseptische Lockerung des Schaftes tritt zwischen 5 und 27\% der Fälle im Bereich der unteren Extremität auf. In diesem Fall sollte eine frühzeitige Revision angestrebt werden [1-3]. Schaftfrakturen sind aufgrund der verbesserten Materialien selten geworden. In der Literatur werden sie zwischen 1 und $22 \%$ angegeben [1-3]. Beim MUTARS-System sind in unserer Klinik keine Schaftfrakturen bei Schäften ab einer Größe von $12 \mathrm{~mm}$ aufgetreten. Der erhältliche zementierte 11-mm-Schaft sollte nur in Ausnahmefällen verwendet werden, da es hier in der Vergangenheit in einigen Fällen zu Schaftfrakturen gekommen ist [1].

Ein Polyethylenverschleiß ist nicht als Komplikation, sondern eher als eine erwartete Abnutzung bei sehr aktiven Patienten anzusehen. Ein zunehmender Verschleiß des Polyethylens führt zu einer zunehmenden Instabilität des betroffenen Gelenks. Des Weiteren kann durch den Polyethylenabrieb eine aseptische Lockerung hervorgerufen werden. Daher sollte ein frühzeitiger Wechsel dieser Verschleißteile durch einen kleinen Eingriff erfolgen.

Die häufigste Komplikation des proximalen Humerusersatzes ist die Dislokation, bedingt durch die Resektion der Rotatorenmanschette und Teile des $\mathrm{M}$. deltoideus. Diese kann durch die Refixation der verbleibenden Muskulatur am Anbindungsschlauch reduziert werden [1-3].

Die Luxationsrate des proximalen Femurersatzes wird in der Literatur mit bis zu 20\% angegeben [4]. In unserer Klinik konnte die Luxationsrate durch die Verwendung eines Anbindungsschlauchs mit Refixation der umliegenden Kapsel und Muskelanteile und eines Duokopfs auf $1,7 \%$ reduziert werden $[1,4]$. Bei Patienten, bei denen eine Pfannenversorgung erforderlich ist, wird derzeitig eine tripolare Pfanne verwendet. Dadurch konnte die Luxationsrate reduziert werden.

Luxationen beim proximalen Femurersatz können durch die Verwendung eines Duokopfs mit Anbindungsschlauch oder der Verwendung einer tripolaren Pfanne reduziert werden.

Beim proximalen Tibiaersatz kann es zu einer Schwäche der aktiven Streckung des Kniegelenks kommen. Durch den Einsatz des MUTARS-Anbindungsschlauchs können insgesamt sehr gute Ergebnisse erzielt und das aktive Streckdefizit deutlich verbessert werden.

\section{Indikationen und Limits}

Die ständige Verbesserung des Prothesenmaterials und der chirurgischen Techniken führt zu einer ständig steigenden Anzahl von Patienten, die extremitätenerhaltend mit modularen Tumormegaendoprothesen operiert werden können.

Eine Gefäß-Nerven-Beteiligung durch den Tumor ist oftmals eine Kontraindikation für den Extremitätenerhalt. Jedoch auch in diesen Fällen ist oft eine ablative Therapie vermeidbar. Mithilfe von modularen Tumormegaendoprothesen und einem Gefäßersatz können gute onkologische und funktionelle Ergebnisse erreicht werden. Eine weitere relative Kontraindikation zur Verwendung einer Tumormegaendoprothese ist die Beteiligung des Streckapparats, im Besonderen bei Tumoren im Bereich des Kniegelenks. Bei einem intraartikulären Tumorbefall des Kniegelenks ist eine Resektion ohne Eröffnung desselben notwendig. Bei einer solchen exartikulären Resektion sollten zumindest Teile des Streckapparats erhalten bleiben. Anderenfalls sind eine Weichteildeckung des Implantats und eine gute Funktion nicht möglich. Falls eine nicht ausreichende Stabilität des Kniegelenks durch den verbliebenen Streckapparat erreicht werden kann, ist eine weitere Stabilisierung durch eine Orthese möglich $[1,4]$.

\section{Funktionelles Ergebnis}

Die funktionellen Ergebnisse nach einer Rekonstruktion mittels einer Tumorprothese sind vielversprechend und können mit dem Musculoskeletal Society Score
(MSTS) gut beurteilt werden [8]. Dazu werden einerseits subjektive Parameter (Zufriedenheit, Schmerzen usw.) als auch funktionelle Parameter (Bewegungsumfang, Hilfsmittel, Gehstrecke usw.) erfasst. $100 \%$ bedeutet, dass der Patient keinerlei Einschränkungen hat. In der Literatur variieren die Ergebnisse zwischen 60 und 90\% [2]. Wir konnten die besten Resultate im Bereich des proximalen Tibiaersatzes (83\%), gefolgt vom distalen Femurersatz (80\%) erreichen. Patienten mit einem proximalen Femurersatz erreichten ein durchschnittliches Ergebnis von $70 \%$. Besonders bei älteren Patienten verbleibt oftmals ein Trendelenburg-Hinken. Viele benötigen ebenfalls eine Gehhilfe auf der gesunden Seite. Patienten mit einem proximalen Humerusersatz erreichten ein durchschnittliches Ergebnis von 70\%. Dies war v.a. durch die schlechte aktive Beweglichkeit des Schultergelenks bedingt, die durch die notwendige Resektion der Rotatorenmanschette resultiert. Die meisten Patienten können den Arm unter starken Anstrengungen nur bis $60^{\circ}$ antevertieren und $30^{\circ}$ abduzieren. Alle Patienten waren jedoch in der Lage, die Hand zum Mund zu führen [1].

\section{Tumorprothesen bei Kindern}

Während die Rekonstruktion mit modularen Tumorendoprothesen bei Erwachsenen Mittel der Wahl ist, kann dies nicht direkt auf Kinder übertragen werden. Die Kombination aus resultierender Beinlängendifferenz während des Wachstums und die Schwierigkeit bei der Teilnahme an Rehabilitationsprogrammen verhindern ähnlich gute Resultate wie bei Jugendlichen und Erwachsenen. Wachstumsprothesen sollten erst bei einer resultierenden Beinlängendifferenz von $4-5 \mathrm{~cm}$ verwendet werden. Bei sehr kleinen Kindern ist eine ablative Therapie, einschließlich der Umdrehplastik, ein übliches Verfahren, da es nicht mit relevanten chirurgischen Problemen vergesellschaftet ist. Als primäres Verfahren konnten mit der Umdrehplastik gute funktionelle Ergebnisse erreicht werden [9]. Jedoch ist jede Art einer Amputation ein mutilierendes Verfahren. Speziell bei Kindern in der Pubertät ist der stigmatisierende Effekt nicht zu vernachlässigen. Verlängerbare Tumormegaendoprothesen sollten bei Kindern nur sehr vorsichtig und umsichtig aufgrund der resultierenden Beinlängendifferenz und der mehrfachen Operationen zur Elongation der Prothese verwendet werden. 
Tumorwachstumsprothesen sollten $a b$ einer resultierenden Beinlängendifferenz von 5-6 cm eingesetzt werden. Bei sehr kleinen Kindern ist eine Umdrehplastik eine sinnvolle Alternative.

Seit 30 Jahren kommen Wachstumsprothesen zur Anwendung. Ein etabliertes System basiert auf einem Verlängerungsmodul mit einem Schraubmechanismus, wie z.B. der Lewis Expandable Adjustable Prosthesis (LEAP), die 1983 eingeführt wurde, oder später das Howmedica Modular Reconstruction System (HMRS). Die Verlängerung erfolgte über einen kleinen Hautschnitt, durch den der Verlängerungsmechanismus mittels Schraubenzieher expandiert wurde. Das Stanmore-Verlängerungssystem (Fa. Stanmore Implants, Elstree, Großbritannien) funktionierte, indem im Mittelteil der Wachstumsprothese ein Verlängerungsstück eingebracht wurde. Beide Systeme haben den Nachteil, dass eine operative Intervention mit einer Ad-hoc-Verlängerung notwendig ist. Die maximale Adhoc-Verlängerung der unteren Extremität beträgt $1,5-2 \mathrm{~cm}$, ansonsten kommt es zu Schäden an Nerven und Gefäßen und/oder zu einer deutlichen Bewegungseinschränkung [10].

Neuere Wachstumsprothesendesigns wie die „Phenix Growing Prosthesis“ (Phenix Medical, Paris, Frankreich) oder die neue Generation der HMRS konnten diese Probleme verbessern. Diese beiden Prothesensysteme bestehen aus ineinander gesteckten Hülsen, in denen sich ein vorgespannter Federmechanismus befindet, der durch ein solides Stück Kunststoff arretiert wird. Wenn eine Verlängerung erforderlich ist, wird das Kunststoffstück durch z.B. ein elektromagnetisches Feld erwärmt und dadurch dem Federmechanismus erlaubt, sich zu expandieren, bis sich der Kunststoff wieder abkühlt. Ergänzend sollte im Bereich der nicht betroffenen Epiphysenfuge ein polierter Schaft eingebracht werden, der ein Restwachstum der Wachstumsfuge erlaubt.

Die MUTARS-Xpand verwendet einen ähnlichen Ansatz. In 2 ineinander sitzenden Hülsen ist ein Motor eingebettet. Durch einen subkutan gelegenen Transmitter kann mithilfe von elektromagnetischen Wellen transkutan Energie zum Ausfahren des Motors übertragen werden. Im Bereich des Oberschenkels kann so eine Verlängerung von $1 \mathrm{~mm} /$
Tag und im Bereich des Unterschenkels von $0,8 \mathrm{~mm} /$ Tag erfolgen. Je nach Prothesenmodell ist eine Verlängerung bis $\mathrm{zu}$ $10 \mathrm{~cm}$ möglich. Eine weitere neue Methode ist die MUTARS-Bioxpand. Dieses Verlängungerungssystem basiert auf dem Prinzip der Kallotasis. Ist eine ausreichende Beinlängendifferenz erreicht, wird eine Verlängerung nach vorheriger Osteotomie durch einen motorisierten Verlängerungsmarknagel (Fitbone-Nagel, Fa. Wittenstein, Igersheim) erreicht. Hier wird im Gegensatz zu herkömmlichen Verlängerungsprothesensystemen nicht die Prothese, sondern der Knochen verlängert. Dadurch kann das Hebelverhältnis zwischen Knochen und Prothese zugunsten des Knochens verbessert werden. Dies soll einerseits zu einer längeren Standzeit des betroffenen Schaftes führen und im Falle eines notwendigen Schaftwechsels genügend verbliebenes Knochenlager bieten. In beiden Fällen kann der Patient in der Regel in ambulanter Behandlung verbleiben. Da beide Prothesendesigns nicht für die aufkommenden Kräfte bei größeren Jugendlichen und Erwachsenen geeignet sind, sollte die Verlängerungsprothese nach Abschluss des Wachstums gegen eine konventionelle getauscht werden.

\section{Zusammenfassung}

Extremitätenerhaltende Chirurgie mit modularen Tumormegaendoprothesen ist zum Standardverfahren mit guten Ergebnissen geworden. Jedoch besonders bei jungen aktiven Patienten, die das ,typische“ Kollektiv der Tumorpatienten darstellen, gerät das Material an seine physikalischen Limits. Eine Revision der Polyethylenverschleißteile ist meistens unvermeidlich. Die schwerwiegendste Komplikation neben dem Lokalrezidiv (1-9\%) ist die periprothetische Infektion (5-36\%) mit dem Risiko der Amputation. Mithilfe der Silberbeschichtung der MUTARS-Prothese konnte beim proximalen Femur- und Tibiaersatz die Infektionsrate auf 5,9\% reduziert und bisher sekundäre Amputationen vermieden werden.

\section{Literatur}

${ }^{1}$ Gosheger G, Gebert C, Ahrens $H$ et al. Endoprosthetic reconstruction in 250 patients with sarcoma. Clin Orthop Relat Res 2006; 450: 164-171

2 Balke M, Ahrens H, Streitburger A et al. Modular endoprosthetic reconstruction in malig- nant bone tumors: indications and limits. Recent Results Cancer Res 2009; 179: 39-50

3 Zeegen EN, Aponte-Tinao LA, Hornicek FJ et al. Survivorship analysis of 141 modular metallic endoprostheses at early followup. Clin Orthop Relat Res 2004; 420: 239-250

4 Bickels J, Meller I, Henshaw RM et al. Reconstruction of hip stability after proximal and total femur resections. Clin Orthop Relat Res 2000; 375: 218-230

5 Picci P, Sangiorgi L, Rougraff BT et al. Relationship of chemotherapy-induced necrosis and surgical margins to local recurrence in osteosarcoma. J Clin Oncol 1994; 12: 2699-2705

6 Grimer RJ, Belthur M, Chandrasekar C et al. Two-stage revision for infected endoprostheses used in tumor surgery. Clin Orthop Relat Res 2002; 395: 193-203

7 Hardes J, von Eiff C, Streitbuerger A et al. Reduction of periprosthetic infection with silver-coated megaprostheses in patients with bone sarcoma. J Surg Oncol 2010; 101: 389395

${ }^{8}$ Enneking WF. A system of staging musculoskeletal neoplasms. Instr Course Lect 1988; 37: 3-10

9 Hillmann A, Hoffmann C, Gosheger G et al. Malignant tumor of the distal part of the femur or the proximal part of the tibia: endoprosthetic replacement or rotationplasty. Functional outcome and quality-of-life measurements. J Bone Joint Surg [Am] 1999; 81: 462-468

10 Babyn PS, Wihlborg CE, Tjong JK et al. Local complications after limb-salvage surgery for pediatric bone tumours: a pictorial essay. Can Assoc Radiol J 2001; 52: 35-42

\section{Dr. med. Ralf Dieckmann}

Assistenzarzt

Dr. med. Arne Streitbuirger

Oberarzt

Dr. med. Marcel-Philipp Henrichs

Assistenzarzt

Markus Nottrott, PhD

Funktionsoberarzt

Univ.-Prof. Dr. med. Georg Gosheger

Klinikdirektor

Priv.-Doz. Dr. med. Jendrik Hardes

Sektionsleiter Tumor- und

Revisionschirugie

Klinik und Poliklinik für Allgemeine

Orthopädie und Tumororthopädie

Universitätsklinikum Münster

Albert-Schweitzer-Straße 33

48149 Münster

ralf.dieckmann@ukmuenster.de

\section{Dr. med. Maurice Balke}

Assistenzarzt

Klinik für Unfallchirurgie, Orthopädie und Sporttraumatologie

Krankenhaus Merheim

Ostmerheimer Straße 200

51109 Köln 